\title{
Selective firing of rat pontine gigantocellular neurons during movement and REM sleep
}

\author{
ROBERT P. VERTES
}

Department of Physiology, University of Michigan Medical School, Ann Arbor, Mich. 48109 (U.S.A.)

(Accepted February 17th, 1977)

Neurons in the gigantocellular field of the pontine reticular formation (PGF) fire at very slow rates during waking (W) and slow wave sleep (SWS) and increase their discharge as much as 100 times during selective segments of the rapid eye movement (REM) sleep cycle ${ }^{8,12}$. Hobson has proposed that these neurons form a center for the control of the various phasic and/or tonic events that are specific to REM sleep? This hypothesis is supported by the observations that lesions within the pontine reticular formation eliminate elements of REM sleep ${ }^{2,6}$ and that prolonged electrical stimulation in this region increases the total amount of REM sleep ${ }^{5,15}$.

The data of Hobson and co-workers ${ }^{8,12}$ were taken from recordings in restrained cats. In a preliminary report in the partially restrained cat, it was demonstrated that PGF neurons that fire at high rates during REM show an equally increased rate of discharge during waking with head movements ${ }^{13}$. In the awake, freely moving rat it was incidentally observed that a very large percentage of PGF neurons show markedly increased activity during movement ${ }^{23}$. If these movement-correlated cells also show the pattern of selective REM-associated activity described by Hobson et al. ${ }^{8}$, the significance of increased PGF activity in REM would require reinterpretation.

Our data were taken from 15 unrestrained male Sprague-Dawley rats (350-450 g) prepared with chronically implanted cervical electromyogram (EMG), bipolar dorsal hippocampal and cortical (skull screw) recording electrodes. A skull-mounted microdrive advanced a metal microelectrode for recording from PGF neurons ${ }^{22}$. After a 5-7-day recovery period, each rat was sleep-deprived overnight by the inverted flower pot method ${ }^{\mathbf{1 6}}$; recording began on the following day.

The microelectrode was advanced in increments less than $30 \mu \mathrm{m}$ through successive cerebellar Purkinje cell layers which were identified by a high frequency, high amplitude multiunit response. Entry into the dorsal pons was marked by a significant reduction in spontaneous cell activity, and single, well isolated, large amplitude spikes were encountered approximately $1 \mathrm{~mm}$ ventral to that point. From that location ventrally any neuron that had an extracellular action potential of $250 \mu \mathrm{V}$ or greater was considered a PGF cell and recorded from. The only criteria, then, used in the selection of cells were that they be localized to the PGF region of the brain stem and that they 
have a large action potential, i.e. $\geq 250 \mu \mathrm{V}$. The method for recording cellular activity was similar to that previously described ${ }^{23}$. Action potentials were displayed on an oscilloscope and relayed to a counter, the output of which was displayed on a polygraph along with the EEG, neck muscle EMG, hippocampal slow waves, and a motion indicator.

Cell activity was counted and compared during the following four behavioral states: (1) waking with movement (W-M); (2) waking without gross movement (W); (3) slow wave sleep (SWS), and (4) cortical desynchronized or REM sleep. These four distinct states were identified by physiological criteria specific for each state. These were: W-M, low voltage, high frequency (LVHF) EEG, hippocampal theta activity, neck muscle EMG; W, LVHF EEG, no hippocampal theta activity, but no large amplitude hippocampal sleep waves, lower amplitude neck muscle EMG than during W-M; SWS, HVLF EEG, large amplitude hippocampal waves, minimal neck EMG; REM, LVHF EEG, hippocampal theta activity, minimal neck EMG. (The slow rhythmic theta activity in the hippocampus is present in the rat during active, voluntary movements and REM sleep ${ }^{21,24}$.) Movements were detected using the 'free wire' technique ${ }^{14}$. Rats were continually observed and notations regarding their behavior were made. For most cells, the sequence of recording was from waking (W and W-M) to sleep (SWS and REM) to waking again. When localizing cells during waking it is conceivable that some neurons were overlooked that had absolutely no spontaneous rate of activity during W. Neurons of this type, however, would appear to be quite distinct from those recorded by Hobson et al. ${ }^{8}$, since the mean spontaneous rate of firing of their cells during W was approximately $6 / \mathrm{sec}$ (see Fig. 2B). In accord with previous methods ${ }^{8}$, the absolute minimum recording time in any behavioral state for any cell was $60 \mathrm{sec}$. In the present study, however, the cell response rates usually represent samples taken over a 3-5-min period. The unit data was analyzed in 6-sec blocks yielding 10 samples $/ \mathrm{min}$. All cells included in the data analysis were recorded during all four behavioral states and had action potential amplitude fluctuations of less than $20 \%$ during recording. The Prussian blue technique was employed for histological localization of the microelectrode tip.

Eighteen cells in 15 rats were localized to the PGF of the brain stem. Fifteen of those 18 neurons fired significantly more in REM than in either SWS or W as described by Hobson et al. ${ }^{8}$ for cat PGF neurons. However, each neuron also showed a significant increase in firing rate during waking with movement (W-M); in fact, the mean firing rate for these 15 neurons was slightly greater in W-M than during REM.

Fig. 1 shows the response of one of these 15 neurons during W, W-M, SWS, and REM. Note that during W there is little cell activity. This period is marked by a relatively quiescent EMG, low amplitude hippocampal slow waves without theta $(6-12 \mathrm{~Hz})$ activity, and a low voltage high frequency EEG. During W-M, however, when the rat is moving around as indicated by the motion detector, the appearance of the hippocampal theta rhythm and the increase in neck EMG, the cell fires at an extremely high rate. With the onset of SWS, characterized by higher amplitude slow waves in both the hippocampal and EEG records, the cell is quiescent. In REM, denoted by the low amplitude EMG, the desynchronized EEG and the appearance of the prominent theta activity in the hippocampal record, the cell once more fires at a very high rate. 


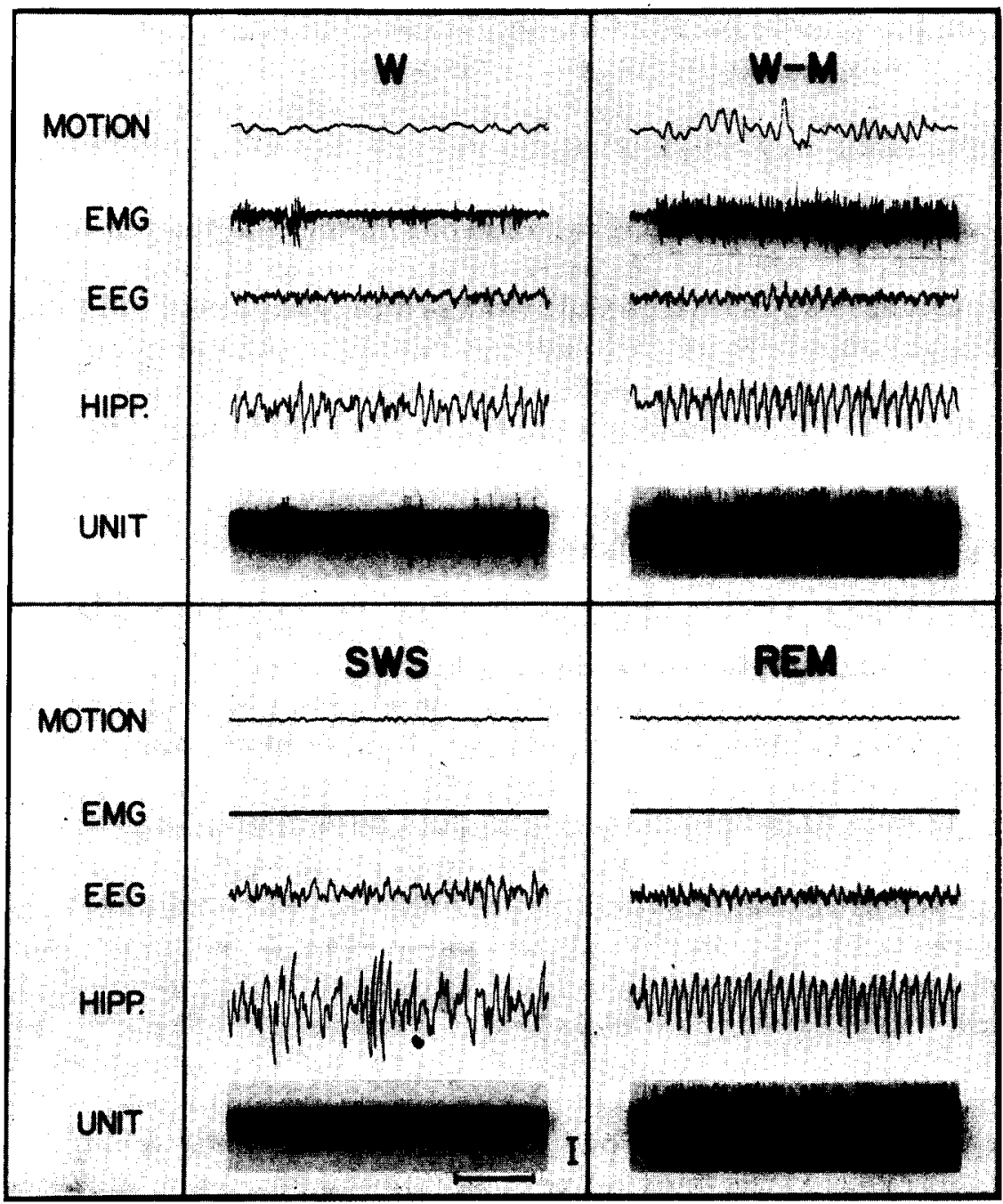

Fig. 1. Firing of a single PGF cell during W, W-M, SWS, and REM along with a motion indicator (first trace), neck EMG, cortical EEG, and hippocampal slow waves, respectively. Note the minimal firing during W and SWS and the greatly increased response during W-M and REM. Calibrations: $1 \mathrm{sec}$ and $100 \mu \mathrm{V}$.

Fig. 2A illustrates the rate of response of each of the 18 cells recorded from during W, W-M, SWS, and REM. The increased rate of response seen both during W-M and REM was highly significant for each of the 15 cells $(P<0.001$, two-tailed $t$-test of mean differences). Most of these cells showed rates in W-M and REM 10-20 times that during $\mathrm{W}$ and SWS. For 7 of the 15 cells the response was higher in W-M than in REM; for the other 8 cells the reverse was true.

Fig. 2B compares the 15 neurons in the present study with 74 neurons found in the PGF region of the cat by Hobson et al. ${ }^{8}$. The differences in cell response between REM and either W or SWS states are more pronounced in the present study than in that 


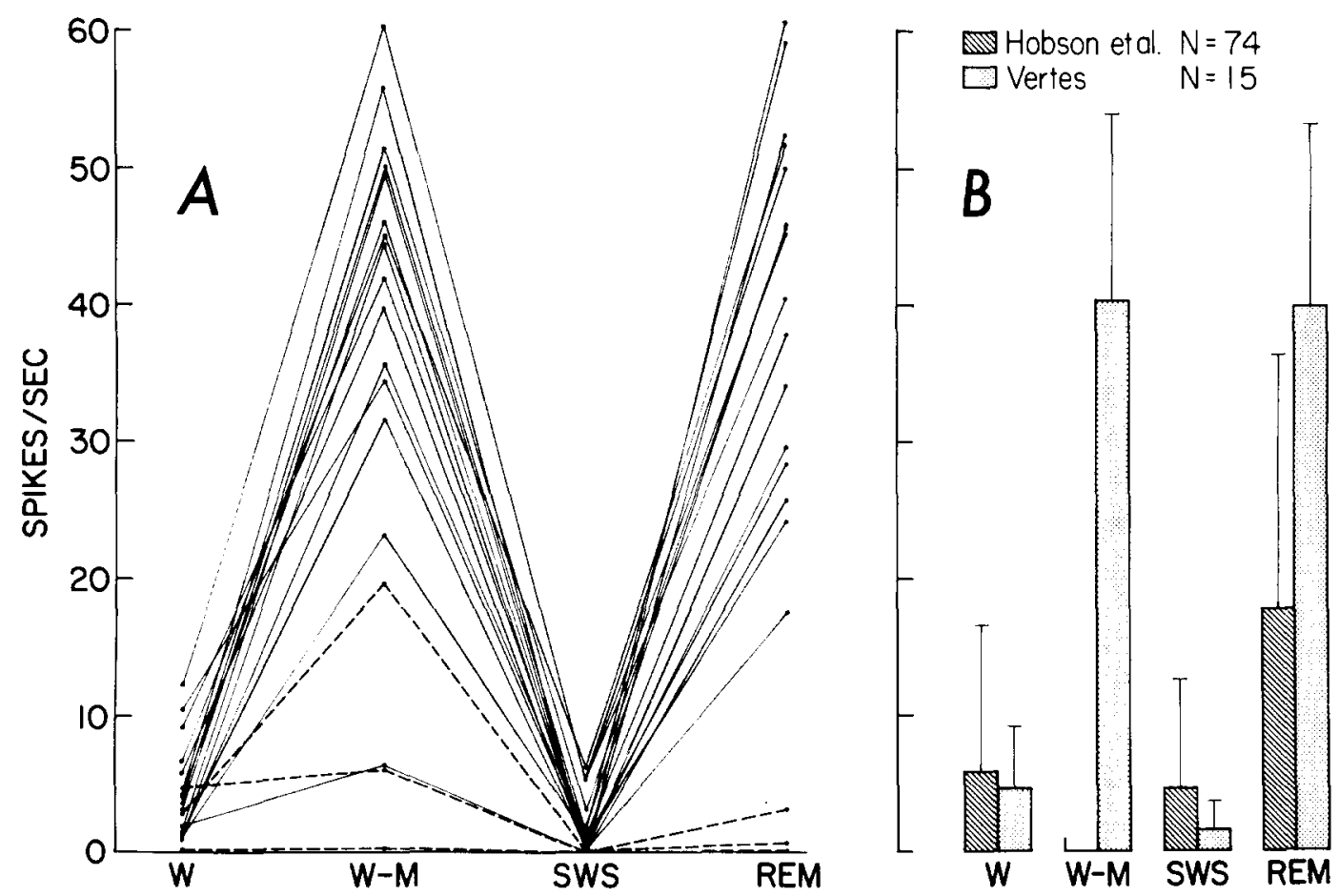

Fig. 2. A: response of 15 PGF neurons (solid lines) that fired significantly more in both W-M and REM than either W and SWS, and 3 PGF cells (dashed lines) that did not. B : comparison of mean firing rates and standard deviations of the $15 \mathrm{~W}-\mathrm{M}-\mathrm{REM}$ responsive neurons of the present study with 74 neurons localized to PGF by Hobson et al. ${ }^{8}$. They did not record during W-M.

of Hobson et al. ${ }^{2}$. A possible explanation could be that their sample of 74 neurons included any cell that could be successfully recorded from the PGF, whereas our analysis includes only those cells showing the characteristic pattern of increased firing during REM ( 15 cells) and excludes those that did not ( 3 cells). This factor could also account for the differences in standard deviations. A comparison of W-M states is not possible since Hobson et al. ${ }^{8}$ did not record during this period.

The schematic sagittal section presented in Fig. 3 shows the rostral-caudal distribution of the neurons from which the present data are drawn. The region sampled spans the nucleus pontis caudalis and pontis oralis ${ }^{20}$. There appears to be no selective grouping between the cells that did respond during W-M and REM and those that did not.

Our results indicate that in the rat, at least, the high frequency discharge of PGF cells is not uniquely associated with the onset or control of REM sleep. It is possible that species and sampling differences have allowed us to record from PGF neurons with properties markedly different from those in the cat. The location and sleep-associated firing rates of our PGF neurons, however, are quite comparable to those of Hobson and co-workers ${ }^{8,12}$. It therefore seems likely that PGF recordings from the unrestrained, moving cat would reveal cells with properties similar to those reported here. Indeed, the pattern of PGF neuron activity is not unlike that found in other regions of the brain. 


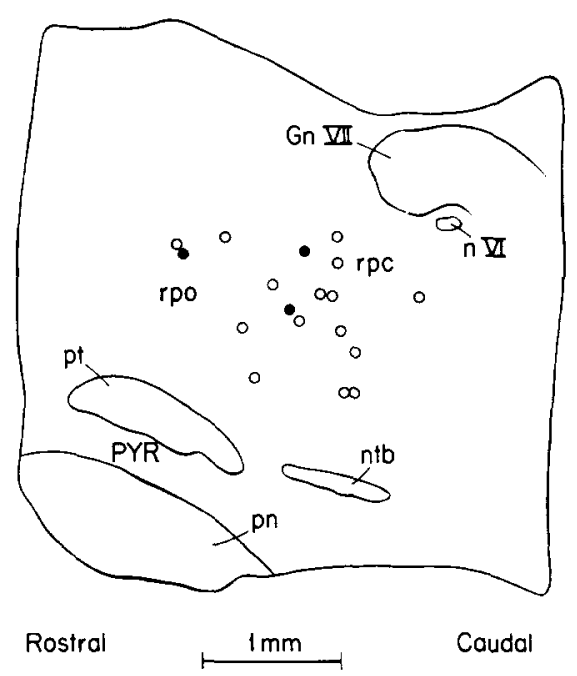

Fig. 3. Schematic sagittal section of the rostral brain stem showing the location of the 15 cells (open circles) that responded during both W-M and REM and 3 cells (closed circles) that did not. All 18 cells were located within the medial-lateral boundaries of the pontine gigantocellular nuclei, i.e. approximately $0.5-1.5 \mathrm{~mm}$ from the midline. Abbreviations: Gn VII, genu of the 7th nerve; $\mathrm{n} \mathrm{VI,} \mathrm{abducens}$ nucleus; ntb, nucleus of the trapezoid body; pn, nucleus of the pons; pt, pontine tegmental nucleus of Bechterev; PYR, pyramidal tract; rpc, nucleus reticularis pontis caudalis; rpo, nucleus reticularis pontis oralis.

In the monkey, Evarts ${ }^{3}$ has shown that cortical neurons projecting in the pyramidal tract decrease their firing rate as the animal passes from W to SWS, and then increase it again in the transition from SWS to REM. These same cells responded significantly more during waking when the animal was moving. Visual cortical neurons also show higher rates of activity during REM and waking with visual stimulation than in either W or SWS ${ }^{4}$. Neurons in the cerebellum, vestibular nuclei, hippocampus, and the midbrain and caudal brain stem reticular formation have all been shown to maintain higher rates of activity during both W-M and REM as compared to W and SWS ${ }^{1,9,11,}$ 17,19 .

The relative magnitude of the increased response of PGF cells during $\mathrm{W}-\mathrm{M}$ and REM is, however, much greater than that described for these other brain regions, suggesting that PGF neurons are involved in some specific function common to both states. High frequency PGF activity, for instance, could be involved in the generation of theta activity in the hippocampus, since both are selectively present during movement and REM sleep. The brain stem reticular formation has long been thought of as the source for the generation of the hippocampal theta rhythm ${ }^{18}$, and PGF electrical stimulation has been shown to produce theta activity in the hippocampus ${ }^{10}$. Alternatively, PGF firing could be related to somatic motor events common to both awake movements and REM sleep. In this regard, as mentioned earlier, it has been demonstrated that cat PGF cells that fire with neck movements during waking also show a significantly increased response during REM sleep ${ }^{13}$. The activity of these neurons, as 
well as some of ours, could be related to the myoclonic twitches of REM sleep and to associated movements during waking.

It is equally possible that identical patterns of neural activity have very different functions in different physiological states of the brain. In any case, it is apparent that the function of these PGF cells in the rat extends beyond the REM sleep state. Forthcoming hypotheses about their function are more likely to be correct if their activity is recorded during a wide variety of behavioral states.

I thank Dr. Kenneth L. Casey for constructive suggestions regarding the research and for his critical reading of this manuscript. I am grateful to Lou Cannella for his excellent assistance.

This work was supported by NIH Fellowship Grant 1 F32 NS05300-01 to Robert P. Vertes, and by NIH Grant 1 R01 NS12015-01 to Kenneth L. Casey.

1 Bizzi, E., Pompeiano, O. and Somogyi, I., Spontaneous activity of single vestibular neurons of unrestrained cats during sleep and wakefulness, Arch. ital. Biol., 102 (1964) 308-330.

2 Carli, G. and Zanchetti, A., A study of pontine lesions suppressing deep sleep in the cat, Arch. ital. Biol., 103 (1965) 751-788.

3 Evarts, E. V., Temporal patterns of discharge of pyramidal tract neurons during sleep and waking in the monkey, J. Neurophysiol., 27 (1964) 152-171.

4 Evarts, E. V., Unit activity in sleep and wakefulness. In G. C. Quarton, T. Melnechuk and F. O. Schmitt (Eds.), The Neurosciences: A Study Program, Rockefeller Univ. Press, New York, 1967, pp. 545-556.

5 Frederickson, C. J. and Hobson, J. A., Electrical stimulation of the brain stem and subsequent sleep, Arch. ital. Biol., 108 (1970) 564-576.

6 Hobson, J. A., The effect of chronic brain stem lesions on cortical and muscular activity during sleep and waking in the cat, Electroenceph. clin. Neurophysiol., 19 (1965) 41-62.

7 Hobson, J. A., The cellular basis of sleep cycle control. In E. Weitzman (Ed.), Advances in Sleep Research, Spectrum, New York, 1974, pp. 217-250.

8 Hobson, J. A., McCarley, R. W., Pivik, R. T. and Freedman, R., Selective firing by cat pontine brain stem neurons in desynchronized sleep, J. Neurophysiol., 37 (1974) 497-511.

9 Huttenlocher, P. R., Evoked and spontaneous activity in single units of medial brain stem during natural sleep and waking, J. Neurophysiol., 24 (1961) 451-468.

10 Macadar, A. W., Chalupa, L. M. and Lindsley, D. B., Differentiation of brain stem loci which affect hippocampal and neocortical electrical activity, Exp. Neurol., 43 (1974) 499-514.

11 Marchesi, G. F. and Strata, P., Mossy and climbing fiber activity during phasic and tonic phenomena of sleep, Pflügers Arch. ges. Physiol., 323 (1971) 219-240.

12 McCarley, R. W. and Hobson, J. A., Single neuron activity in cat gigantocellular tegmental field: selectivity of discharge in desynchronized sleep, Science, 174 (1971) 1250-1252.

13 McGinty, D. J. and Fairbanks, M. K., Discharge pattern in gigantocellular pontine neurons: comparison of waking and REM sleep, Neurosci. Abstracts, 1 (1975) 489

14 Mink, W. D., Best, P. J. and Olds, J., Neurons in paradoxical sleep and motivated behavior, Science, 158 (1967) 1335-1337.

15 Monti, J. M., Effect of recurrent stimulation of the brain stem reticular formation on REM sleep in cats, Exp. Neurol., 28 (1970) 484-493.

16 Morden, B., Mitchell, G. and Dement, W., Selective REM sleep deprivation and compensation phenomena in the rat, Brain Research, 5 (1967) 339-349.

17 Noda, H., Manohar, S. and Adey, W. R., Spontaneous activity of cat hippocampal neurons in sleep and wakefulness, Exp. Neurol., 24 (1969) 217-231.

18 Petsche, H., Gogolak, G. and Van Zwieten, P. A., Rhythmicity of septal cell discharges at various levels of reticular excitation, Electroenceph. clin. Neurophysiol., 19 (1965) 25-33.

19 Ranck, J. B., Jr., Studies on single neurons in dorsal hippocampal formation and septum in unrestrained rats. I. Behavioral correlates and firing repertoires, Exp. Neurol., 41 (1973) 461-531 
20 Valverde, F., Reticular formation of the albino rat's brain, cytoarchitecture and corticofugal connections, J. comp. Neurol., 119 (1952) 25-53.

21 Vanderwolf, C. H., Hippocampal electrical activity and voluntary movement in the rat, Electroenceph. clin. Neurophysiol., 26 (1969) 407-418.

22 Vertes, R. P., A device for recording single unit activity in freely-moving rats by a movable finewire microelectrode, Electroenceph. clin. Neurophysiol., 38 (1975) 90-92.

23 Vertes, R. P. and Miller, N. E., Brain stem neurons that fire selectively to a conditioned stimulus for shock, Brain Research, 103 (1976) 229-242.

24 Winson, J., Interspecies differences in the occurrence of theta, Behav. Biol., 7 (1972) 479-487. 